\title{
Jornal Brasileiro de Pneumologia: 40 years of tradition
}

\author{
Nelson Morrone \\ Editor-in-Chief of the Journal of Pulmonology from 1990 to 1994.
}

For humans, turning forty is almost a cause for despair, because it signals the end of youth as well as a less brilliant future. For a medical journal, however, 40 years of existence means that it has had a satisfactory history, and that, more importantly, it will have an increasingly bright future.

The Brazilian Journal of Pulmonology (BJP), which is celebrating its 40th anniversary, stands out today for a number of reasons, chief among which is that it disseminates research conducted by Brazilian and international experts. This internationalization reflects the maturity and dissemination power of the Journal. One important contribution to that internationalization, as well as to attracting researchers working in Brazil, was our acceptance for indexing by LILACS, PubMed/MEDLINE, and other databases. The online publication of all articles, in Portuguese and in English (in-house translation by the $\mathrm{BJP}$ ), in parallel with the publication of a print version, in Portuguese, English, or Spanish, depending on the language of submission, is an invaluable achievement and confers considerable prestige. Changing the name of our Journal from the Jornal de Pneumologia (Journal of Pulmonology) to the Jornal Brasileiro de Pneumologia (Brazilian Journal of Pulmonology) undoubtedly made a considerable contribution to the prestige it currently enjoys.

Another truly important aspect of the BJP is that, unlike many publications, its online version is available for free. It therefore differs from other scientific journals in that it is not a source of revenue for the professional society that sponsors it-in our case the Brazilian Thoracic Association. This is clearly an important contribution to medical knowledge, with obvious advantages for patients, distinguishing our Journal from those that charge a fee for viewing an article.

The greatness of the BJP reflects the fact that numerous initial difficulties were surmounted, many by progress in general and other by advances in the field of information technology. For example, I remember that the government financed the publication of our Journal by providing an annual stipend, which was constantly devalued by inflation, and that we were prohibited from investing those funds. The reporting of expenditures was an onerous chore, given that even postage stamps had to be accounted for (a point of curiosity: a few years after leaving the $B J P$, I was fined by the tax authority because $R \$ 3.00$, which is currently equivalent to less than US\$1.00, had not been accounted for). This nuisance ended when we began to purchase a year's worth of printing paper all at once. Selling advertising space usually required the collaboration of several colleagues who had access to laboratories, given that the laboratories had little faith in the dissemination power of the BJP. It also should be noted that advertisements were not (and still are not) placed amid the scientific articles in the BJP.

The process of reviewing articles for publication was quite cumbersome, given that the articles were physically delivered to the Journal as hard copies, in at least quadruplicate (originals or photocopies), which then had to be mailed to at least three reviewers. In general, the review process took a few months per article. Today, it takes much less time. Long live the Internet!

The BJP was not always the journal of choice for Brazilian researchers wanting to publish their papers. Therefore, the availability of articles was not always ideal. To overcome that difficulty in the early days, considerable space was allocated to the publication of continuing education pieces, in various sections, in successive issues. However, the failure of some invited authors to meet their deadlines had a negative impact on the periodicity of the BJP. It was therefore decided that review articles would be written mostly by invitation, although unsolicited review articles were also welcome, and would be published in a single issue.

After the material had been fully reviewed and approved, it also took it a few weeks to be published because of technical difficulties involving the printing company the Journal was using at the time. Mailing was another cause of considerable delay. I get chills when I remember that, at the time, one of the directors (Dr. Bogossian) would spend hours at the post office.

The difficulties that have been overcome, some during my time at the journal and some during the terms of subsequent editors, are justification enough for continuing the tradition that is the BJP. 